\title{
Dynamic aspects of pressure and temperature-stabilized intermediates of outer surface protein A
}

\author{
Takuro Wakamoto $^{1}$, Soichiro Kitazawa ${ }^{1}$, Tomoshi Kameda ${ }^{2}$, and Ryo Kitahara ${ }^{3}$ \\ ${ }^{1}$ Ritsumeikan Daigaku - Biwako Kusatsu Campus \\ ${ }^{2}$ National Institute of Advanced Industrial Science and Technology Tokyo Bay Area Center \\ ${ }^{3}$ Ritsumeikan University - Biwako Kusatsu Campus
}

April 28, 2020

\begin{abstract}
Structural characterization of alternatively folded and partially disordered protein conformations remains challenging. Outer surface protein A (OspA) is a pivotal protein in Borrelia infection, which is the etiological agent of Lyme disease. OspA exists in equilibrium with intermediate conformations, in which the central and the C-terminal regions of the protein have lower stabilities than the N-terminal. Here, we characterize pressure- and temperature-stabilized intermediates of OspA by nuclear magnetic resonance spectroscopy combined with paramagnetic relaxation enhancement (PRE). We found that the C-terminal region of the intermediate was partially disordered; however, it retains weak specific contact with the N-terminal region, owing to a twist of the central $\beta$-sheet and increased flexibility in the polypeptide chain. The disordered C-terminal region of the pressure-stabilized intermediate was more compact than that of the temperature-stabilized form. Further, molecular dynamics simulation demonstrated that temperature-induced disordering of the $\beta$-sheet was initiated at the C-terminal region and continued through to the central region. An ensemble of simulation snapshots qualitatively described the PRE data from the intermediate and indicated that the intermediate structures of OspA may expose tick receptor-binding sites more readily than does the basic folded conformation.
\end{abstract}

\section{Introduction}

Proteins in solution fluctuate between folded and unfolded conformations, which is often related to their functions and toxic aggregate formation. ${ }^{1-4}$ Outer surface protein A (OspA) is an immunogenic lipoprotein expressed by the spirocheteBorrelia (e.g. B. burgdorferi, B. garinii, and B. afzelii), the etiologic agent of Lyme disease. It consists of 21 repeated $\beta$-strands and a short C-terminal $\alpha$-helix. OspA also contains two globular domains, the N-terminal $(\beta 1-\beta 7)$ and the C-terminal ( $\beta 11-\mathrm{C}$-terminus) domains, as well as a single-layer $\beta$-sheet (central $\beta$-sheet, $\beta 8-\beta 10$ ) that connects the terminal domains. ${ }^{5,6}$

OspA binds to the tick receptor TROSPA when it enters the tick gut. ${ }^{7}$ Since the receptor-binding sites are occluded in the interior of the C-terminal domain, ${ }^{8}$ their exposure is believed to be required for receptor-binding. Koide et al employed native state hydrogen exchange nuclear magnetic resonance (NMR) spectroscopy to reveal that the C-terminal region, more specifically, the $\beta 9$-C-terminus, including a portion of the single-layer $\beta$-sheet and the C-terminal domain, of this protein is less stable than the N-terminal domain. This study provided the first evidence of disordered intermediate conformations in the C-terminal regions of the protein. ${ }^{9-11}$ They also reported that the intermediate became stabilized as temperature increased. ${ }^{12,13}$ The temperaturestabilized intermediate was characterized using solution NMR, ${ }^{12}$ differential scanning calorimetry, ${ }^{12,13}$ and small angle X-ray scattering. ${ }^{11}$ Moreover, our group found that the pressure-stabilized intermediate was nearly fully disordered in the entire C-terminal region of the polypeptide chain, from $\beta 9$ to the C-terminus. The formation of this intermediate was caused by exposure of a large internal cavity in the C-terminal 
domain. ${ }^{14}$ Recently, Makabe et al reported similar identity between a kinetic intermediate and the equilibrium intermediate. ${ }^{15}$ These results indicate that the pressure- and temperature-stabilized intermediates have similar structural characteristics to those existing under physiological conditions.

Transition into the intermediate in vivo may accelerate the binding of OspA to the tick receptor, as the intermediate exposes the receptor-binding sites (i.e. residues $236-237$ and $242-244)^{8}$, and can interact with a receptor distant from OspA. ${ }^{14}$ In addition, the intermediate may be important for efficient translocation of the protein through the outer membrane. ${ }^{16}$ More importantly, OspA and its C-terminal fragment have been included as vaccine candidates to prevent Borreliatransmission, ${ }^{17}$ some of which have been tested in Phase 3 clinical trials. ${ }^{18}$ A recombinant vaccine using B. burgdorferi OspA was previously licensed for Lyme disease (LYMErix, SmithKlineBeecham, Pittsburgh, PA, USA) in 1998, however, the manufacturer voluntarily withdrew the product from the market 3 years later. ${ }^{19}$ Although human vaccines for Lyme disease are not currently available, genetically modified OspA, which contain protective elements from two different OspA serotypes, continue to represent important vaccine candidates. ${ }^{18}$

Although previous studies have reported that the C-terminal half of the protein ( $\beta 9$-C-terminus) exhibits lower stability than the $\mathrm{N}$-terminal half, our understanding of how the central $\beta$-sheet and $\mathrm{C}$-terminal domain are disordered remains limited. Here, we further characterized the pressure- and temperature-stabilized intermediates of OspA using a paramagnetic relaxation enhancement (PRE)-assisted NMR. ${ }^{20-24}$ The PRE effect arises from dipole-dipole interactions between unpaired electrons of the paramagnetic agent and the nucleus of interest, and thus, spin relaxation contributions show $r^{-6}$ dependence of distance between the paramagnetic center and nucleus. Accordingly, when the paramagnetic agent was conjugated to a portion of the central domain, we were able to collect information on the distance between the central $\beta$-sheet and the $\mathrm{N}$ - and C-terminal domains.

\section{Materials and Methods}

\section{Sample preparation}

A soluble form of OspA, ${ }^{25}$ consisting of residues 18-273 whereby the membrane-associated N-terminal region (residues 1-6) was truncated and the cysteine at residue 84 was substituted by serine, was used as the pseudo wild-type (C84S; WT*) form of OspA (BMRB Entry 4076). Uniformly ${ }^{15}$ N-labeled OspA WT* and three variants (D118C, E128C, and A140C) were produced by conventional E. coli expression in M9 medium with ${ }^{15} \mathrm{NH}_{4} \mathrm{Cl}$ as the sole nitrogen source. The protein was purified by a $\mathrm{Ni}^{2+}$-affinity column (Bio-Rad Laboratories, Hercules, CA) and a HiLoad 26/60 Superdex 75 prep grade column in the AKTA explore 10S (GE Healthcare Life Sciences, Pittsburgh, PA). To cleave the His-tag, the protease thrombin (GE Healthcare Life Sciences) was used. The cysteine variants were dissolved in $20 \mathrm{mM}$ Tris-HCl buffer at $\mathrm{pH} 7.0$, mixed with $S$-([1-oxyl-2,2,5,5-tetramethyl-2,5-dihydro-1H-pyrrol-3-yl]methyl) methanesulfonothioate (MTSL) (Toronto Research Chemicals, Ontario, Canada), and incubated for approximately $12 \mathrm{~h} \mathrm{in} 20 \mathrm{mM}$ Tris-HCl buffer at $277 \mathrm{~K}$. The protein solution was filtered and concentrated using a Microcon (Merck Millipore, Billerica, MA, USA). The final protein solution was adjusted to a concentration of $0.3 \mathrm{mM}$ for PRE experiments in $10 \mathrm{mM}$ phosphate buffer ( $\mathrm{pH} 5.9$ ) containing $50 \mathrm{mM} \mathrm{NaCl}$ and $10 \%{ }^{2} \mathrm{H}_{2} \mathrm{O}$. The protein solution for temperature experiments contained $0.4 \mathrm{M}$ guanidium chloride $(\mathrm{GdmCl})$ in phosphate buffer. The spin-labeled MTSL was reduced with a 2-fold excess of ascorbic acid relative to the protein concentration.

\subsection{NMR experiments}

High-pressure NMR experiments were performed on an AVANCE3-950 $\left({ }^{1} \mathrm{H}, 950.33 \mathrm{MHz}\right)$ spectrometer (Bruker, Billerica, MA, USA) with a cryo-probe system using a pressure-resistant NMR cell (Daedalus Innovations, Aston, PA, USA). For temperature-variable experiments, the slot NMR tube for high-concentration electrolyte samples (SHIGEMI, Hachioji, Japan) was used. To measure PRE ${ }^{1} \mathrm{H} /{ }^{15} \mathrm{~N}$ heteronuclear singlequantum correlation (HSQC) spectra of the MTSL-bound OspA and its variants were collected for the oxidized and reduced forms of MTSL at multiple pressures and temperatures. A 200 complex $\times 4096$ real data matrix was acquired with spectral widths of $36.0\left({ }^{15} \mathrm{~N}, F_{1}\right)$ and $12.98 \mathrm{ppm}\left({ }^{1} \mathrm{H}, F_{2}\right)$. NMR data were processed using Topspin version 3.2 (Bruker, Billerica, MA, USA), NMRPipe, ${ }^{26}$ and NMRViewJ. ${ }^{27}{ }^{1} \mathrm{H}$ che- 
mical shifts were referenced to the methyl signals of 2,2-dimethyl-2-silapentane-5-sulfonate (DSS), and ${ }^{15} \mathrm{~N}$ chemical shifts were indirectly referenced to DSS (0 ppm for $\left.{ }^{1} \mathrm{H}\right)$.

\subsection{Thermodynamic analysis}

The pressure-induced conformational transition from the native state $(\mathrm{N})$ to the intermediate state (I), was determined by examining changes in NMR peak intensities, i.e. peak-area of L109 and V199 methyl groups to estimate an equilibrium constant $K(=[I] /[N])$. Assuming two-state exchange between $\mathrm{N}$ and $\mathrm{I}$, the Gibbs free energy difference at any given pressure $\Delta G^{\mathrm{p}}$ was obtained according to Equation 1 . The Gibbs free energy difference $\Delta G^{0}$ and the partial molar volume difference $\Delta V^{0}$ between the two conformers at $0.1 \mathrm{MPa}$ was estimated by assuming zero compressibility difference between the states as follows:

$G^{p}=-\operatorname{RT} \ln K=G^{0}+V^{0}\left(p-p^{0}\right)$

where $R$ is the gas constant, $T$ is the absolute temperature, $p$ and $p^{0}$ are high pressure and $0.1 \mathrm{MPa}$, respectively.

\subsection{Analysis of PRE}

The ratio of peak height of a paramagnetic sample to that of a diamagnetic sample, that is, $I_{\text {para }} / I_{\text {dia }}$, can be expressed as in Equation 2:22

$\frac{I_{\text {para }}}{I_{\text {dia }}}=\frac{R_{2 i n t} \exp \left(-R_{2 P R E} t\right)}{R_{2 i n t}+R_{2 P R E}}(2)$

where $R_{2 \text { int }}$ and $R_{2 \mathrm{PRE}}$ represent the intrinsic transverse relaxation rate and PRE effects on the transverse relaxation rate for each amide proton, respectively; and $t$ is the total INEPT evolution time of the HSQC $(11.5 \mathrm{~ms})$. The $R_{2 \text { int }}$ for each amide proton was calculated from the half-height line-width of peaks of the diamagnetic sample. Using Equation 2, the $R_{2 \mathrm{PRE}}$ was estimated by assigning the $t$ and $I$ para $/ I_{\text {dia }}$ values. The $R$ 2PRE was then converted into distance using Equation 3:

$r^{6}=\frac{K}{R_{2 P R E}}\left(4 \tau_{c}+\frac{3 \tau_{c}}{1+\varpi_{H}^{2} \tau_{c}^{2}}\right)(3)$

where $r$ is the distance between the unpaired electron and nucleus, $\omega_{\mathrm{H}}$ is the Larmor frequency of protons, $K$ is $1.23 \times 10^{-32} \mathrm{~cm}^{6} \mathrm{~s}^{-2},{ }^{28}$ and $\tau_{\mathrm{c}}$ is the correlation time given as $1 / \tau_{\mathrm{c}}=1 / \tau_{\mathrm{r}}+1 / \tau_{\mathrm{e}}\left(\tau_{\mathrm{r}}\right.$ is the rotational correlation time of the electron-nucleus vector and $\tau_{\mathrm{e}}$ is the electron spin relaxation time). Since $\tau_{\mathrm{e}}(>$ $10^{-7} \mathrm{~s}$ ) of the unpaired electron is much longer than $\tau_{\mathrm{r}}, \tau_{\mathrm{c}}$ is approximately equal to $\tau_{\mathrm{r}}$. The $\tau_{\mathrm{c}}$ value is approximately $12 \mathrm{~ns}$ for the folded conformation of OspA at $318 \mathrm{~K}$ and $0.1 \mathrm{MPa} .{ }^{29}$ The half-height linewidths $(\Delta \nu$ (hertz $\left.)=R_{2 \text { int }} / \pi\right)$ were approximately $20 \mathrm{~Hz}$ for amide proton signals in the protein. The distances from the $I_{\text {para }} / I_{\text {dia }}$ were estimated with a $\tau_{\mathrm{r}}$ of $12 \mathrm{~ns}$ and $R_{2 \text { int }}(20 \mathrm{~Hz})$. Back-calculation of $I$ para $/ I_{\text {dia }}$ for structural models of OspA was performed with $\tau_{\mathrm{r}}=12 \mathrm{~ns}$ and $R{ }_{2 \text { int }}=20 \mathrm{~Hz}$.

Alternatively, estimation of PRE for the unfolded polypeptide chain is not as straightforward due to the fluctuations in both the orientation of the spins and its distance. ${ }^{30}$ Therefore, the distance $r$ was characterized according to the probability distribution. Here, to simulate PREs of the OspA temperature-stabilized intermediate, we used the simplest restraining model, in which $r$ is between the distance of closest approach for the two spheres (i.e. unpaired electron and nucleus). The $r^{6}$ in Eq. 3 can be substituted with $d{ }_{0}^{3} L^{3}$, assuming that the time scale of the distance fluctuation is slow on the time scale of $\tau_{\text {rot }} \cdot{ }^{30} d{ }_{0}$ and $L$ are the distance of the minimum and the maximum separation, respectively. Further, the probability distribution of $r$ was obtained from a trajectory of molecular dynamics simulation for heat unfolding of the protein (seeMolecular dynamics simulation), and $d_{0}$ and $L$ were estimated from the distance distribution. The $\tau_{\mathrm{c}}$ of $12 \mathrm{~ns}$, which is for native state OspA, was used for the intermediate conformations of the protein. More realistic restraining models were discussed in the literature. ${ }^{30}$

\subsection{Molecular dynamics simulation}

Molecular dynamics (MD) simulation of $1 \mu$ s was performed for OspA using the Amber18 simulator ${ }^{31}$ with the AMBER ff14SBonlysc force field ${ }^{31}$ and the generalized born (GB) solvent model. ${ }^{32}$ The crystal structure 
of the protein (PDB:1OSP) was used for the initial conformation. The simulation was conducted with the NVT ensemble, and temperature $(350 \mathrm{~K})$ was controlled using a Langevin thermostat with the solvent viscosity of water to $1.0 \mathrm{ps}^{-1}$. Noncovalent interactions, including electrostatic, van der Waals, and GB, were used without cutoffs. Covalent bonds, including hydrogen atoms, were constrained using the SHAKE method and the integration time step was 2 fs. Snapshots were recorded every $0.1 \mathrm{~ns}$.

\section{Results and Discussion}

\subsection{Pressure- and temperature-induced changes in $^{1} \mathrm{H}-{ }^{15} \mathrm{~N}$ HSQC spectra}

${ }^{1} \mathrm{H} /{ }^{15} \mathrm{~N}$ HSQC spectra of the three variants were measured with the oxidized and reduced forms of MTSL at 0.1, 200, and $250 \mathrm{MPa}$ for $313 \mathrm{~K}$, using an AVANCE3 $950 \mathrm{MHz}$ spectrometer. ${ }^{1} \mathrm{H} /{ }^{15} \mathrm{~N}$ HSQC cross-peaks in the variant proteins were assigned to individual amino-acid residues with reference to the assignments of WT* OspA (BMRB code: 4076). ${ }^{9}$ To evaluate the effects of residue substitution and the MTSL-tag, the ${ }^{1} \mathrm{H}$ and ${ }^{15} \mathrm{~N}$ chemical shifts of individual cross-peaks in the HSQC spectra were compared between the WT* and MTSL-tagged cysteine variants (D118C, E128C, and A140C) at $313 \mathrm{~K}$ and $0.1 \mathrm{MPa}$. Chemical shift changes (i.e. $\left.\left((\Delta \delta \mathrm{H})^{2}+(\Delta \delta \mathrm{N} / 5)^{2}\right)^{0.5}\right)$ are shown in Figure S1. Since chemical shift changes larger than $0.2 \mathrm{ppm}$ were only observed at residues spatially proximal to the substituted residue, they were likely the result of local changes in magnetic shielding, and thus, the effects of residue substitution, and the MTSL-tag on the structure and conformational dynamics of the protein are expectedly limited.

Pressure effects on the ${ }^{1} \mathrm{H} /{ }^{15} \mathrm{~N}-\mathrm{HSQC}$ spectra of the E128C variant reduced form are shown in Figure 2. The HSQC cross-peaks corresponding to the folded conformation were well-dispersed, and the chemical shifts of the backbone amide proton and nitrogen were similar to those of $\mathrm{WT}^{*}$ (Figure S1B); thus, the folded conformation of the variant was similar to that of $\mathrm{WT}^{*}$. Pressure-induced changes in the intensities (i.e., volumes) of the cross-peaks present in the $0.1 \mathrm{MPa}$ spectrum (the original cross-peaks) are plotted in Figure S2. As pressure was increased to $150 \mathrm{MPa}$, the intensities of original cross-peaks showed minimal changes. However, several peaks corresponding to amide groups in the central and C-terminal regions preferentially and gradually decreased when pressure exceeded $150 \mathrm{MPa}$, after which relative intensities reached values of approximately 0.2. Additionally, many new peaks appeared in the central portion of the spectrum, where disordered polypeptide chains are typically observed (Figure 2). These results indicate that the locally disordered, intermediate conformation was partially stabilized under high pressure and occurred within $80 \%$ of the total protein.

Moreover, peak intensities (i.e., volumes) relative to those at atmospheric pressure are plotted along with the residue number at $250 \mathrm{MPa}$ (Figure 3A), indicating that pressure-induced conformational changes occur at residues $\beta 8$ to the C-terminus. A few peak also showed mountain-like intensity profiles (initial gain followed by a decrease in intensity with pressure) similar to those of WT* (Figure S2). As discussed previously ${ }^{14}$, conformational dynamics on the microsecond-to-millisecond time scale may correlate with broadening and missing of the cross-peaks, even at $0.1 \mathrm{MPa}$.

When the temperature was increased from 303 to $318 \mathrm{~K}$ at $0.1 \mathrm{MPa}$, preferential decreases in cross-peaks were observed for residues between $\beta 8$ and the C-terminus (Figure 3B), and relative intensities reached values of approximately 0.2 (Figure S3). Besides, many new peaks appeared in the central aspect of the spectrum (Figure S4), as observed in the pressure experiments.

Similar pressure- and temperature-induced decreases in peak intensities were observed in the ${ }^{1} \mathrm{H}$ NMR spectra for L109 and V199 methyl protons (Figure S5), which are located within the central $\beta$-sheet and C-terminal domain of the variant (data not shown). The transitions of the two side-chain signals, occurring in the same pressure and temperature ranges as those of backbone amides, serve as direct evidence for the cooperative transition of the central $\beta$-sheet and the $\mathrm{C}$-terminal domain into the intermediate state. Based on pressure-induced changes in peak intensities of the methyl groups, the Gibbs free energy difference $(\Delta G$ $\left.{ }^{0}\right)$ and the partial molar volume difference $\left(\Delta V^{0}\right)$ between the two conformers at $0.1 \mathrm{MPa}$ and $313 \mathrm{~K}$ were estimated to be $17 \pm 3 \mathrm{~kJ} / \mathrm{mol}$ and $-80+-12 \mathrm{~mL} / \mathrm{mol}$, respectively, which are slightly smaller than those of WT*. ${ }^{14}$ As discussed previously, the large volume decrease observed by this transition may be the result of 
collapse and hydration of the large hydrophobic cavity in the C-terminal domain (Fig. 1). ${ }^{14,35}$

An interesting observation is that cross-peak intensities of several amide groups in the N-terminal domain were relatively increased when the central and C-terminal domains were denatured under higher pressure (above $150 \mathrm{MPa}$ ) or temperature (higher than $313 \mathrm{~K}$ ) (Figures S2 and S3). Although we did not delve deeper into the issue, a rotational motion of the folded N-terminal domain may have been enhanced, thereby producing more intense signals when the central and C-terminal domains were denatured.

\subsection{Pressure- and temperature-stabilized intermediates}

To further characterize the pressure- and temperature-stabilized intermediates, PRE effects on the ${ }^{1} \mathrm{H}$ transverse relaxation rate constant, $R_{2}$ were measured for the variant. Rather than directly measuring the relaxation rates from a series of two-dimensional NMR experiments, we obtained the ratios of peak heights for a paramagnetic (oxidized) sample to those of a diamagnetic (reduced) sample, i.e., $I_{\text {para }} / I_{\text {dia }}$ (Eq. 2). ${ }^{22}$ Figure $4 \mathrm{~A}$ shows the ratio for each amide group in the E128C variant along with the residue number (i.e., $25-110)$ at 0.1 , and $250 \mathrm{MPa}$ at $313 \mathrm{~K}$. The $I$ para $/ I$ dia of residues from 25 to 275 are shown in Figure S6. When the ratio was 1 , no PRE effects were observed. In contrast, a lower ratio indicated greater PRE effects. Assuming an intrinsic line width at half-height $(\Delta \nu$ (hertz $\left.)=R_{2 \text { int }} / \pi\right)$ of $20 \mathrm{~Hz}$ and correlation time of 12 ns for the folded OspA, distances between the paramagnetic probe and nucleus of interest were calculated by a previously described method (Figure S7, Eq. 3). ${ }^{22}$ Note that the multiplicity of MTSL positions was not taken into account in the calculation. At $0.1 \mathrm{MPa}$, ratios of less than 0.8 , corresponding to a distance of $15^{\sim} 20 \AA$ from the paramagnetic probe, were observed for residues $82,83,101$, and 102, while values $<$ 0.3 corresponded to a distance of $12^{\sim} 15 \AA$ for residues $103-110$. These results indicate that residues $103-$ 110 are closer to residue 128 than they are to residues $82,83,101$, and 102 , consistent with the structure resolved by X-ray crystallography at atmospheric pressure. ${ }^{6}$ At $250 \mathrm{MPa}, I$ para $/ I_{\text {dia }}$ showed both increased and decreased values at several sites. For example, the ratios for residues 82, 83, and 102 were increased by approximately 0.2 , indicating that they drifted away from residue 128. In contrast, a remarkable decrease in the ratio for residue 59 indicated that it shifted toward residue 128. Figure 5A shows the locations of residues $59,62,82,83$, and 128, while the pressure dependence details for the ratios are shown in Figure 5B.

Figure $4 \mathrm{~B}$ shows the intensity ratio, $I_{\text {para }} / I_{\text {dia }}$, for each amide group of the $\mathrm{E} 128 \mathrm{C}$ variant for each residue at 303 and $318 \mathrm{~K}$ at $0.1 \mathrm{MPa}$. As the temperature increased, the ratios of residues 80, 82, and 83 increased by approximately $0.1-0.2$, indicating they moved away from residue 128 . Alternatively, slight decreases in the ratios for residues $33,36,37,59,62$, and 105 indicated that these residues shifted closer to residue 128. Figure $5 \mathrm{C}$ shows the gradual increases and decreases in $I$ para $/ I$ dia of residues $59,62,82$, and 83 as temperature increases.

Different behaviors were observed at residues 36, 37, and 106 between increasing pressure and increasing temperature, as shown in Figures 6 and S8. As pressure increased, split cross-peaks were observed (Figure $6 \mathrm{~A}$ ). The second (new) cross-peak increased at the expense of the first (original) cross-peak with increasing pressure. In contrast, only a single peak was observed as the temperature was increased from 303 to $318 \mathrm{~K}$ (Figure 6B). These observations indicate that these amide groups have at least two folded conformations. Assuming a two-state exchange process, split peaks can be observed if the exchange rate, namely $k_{\text {ex }}$, is much smaller than the chemical shift difference $(\Delta \omega)$ between two states; however, a single averaged peak is observed if $k$ ex is much larger than $\Delta \omega$. Figure $6 \mathrm{C}$ shows the $I$ para $/ I$ dia values of original and new peaks for residues 36 and 37, and 106 at $200 \mathrm{MPa}$, where both original and new peaks were observed in the HSQC spectrum. For residues 36 and 37, new peaks showed $0.2-0.4$-fold smaller values than the original peaks, indicating shorter distances in the intermediate than in the native conformations (Figure S6). Conversely, the new peak of residue 106 demonstrated a 0.6-fold larger value than the original peak, indicating much longer distance in the intermediate than in the native conformation. In the case of the averaged peak, the observed PRE effect is the weighted population average of the PRE effects for the two conformations, i.e., native and intermediate conformations. Since the average $I$ para $/ I$ dia values of residues 36, 37, and 106 at $318 \mathrm{~K}$ and $0.1 \mathrm{MPa}$ are similar to those of their new peaks (200 MPa and $313 \mathrm{~K}$ ) (Figure 6D), the second conformation might be dominantly populated at $318 \mathrm{~K}$. 
Pressure and temperature effects on $I$ para $/ I$ dia for each amide group were also investigated for the variants D118C and A140C, as shown in Figures S9 and S10, respectively. As pressure increased, the $I$ para $/ I$ dia values of residues 59-61 of the D118C variant increased by approximately 0.1-0.2 (Figure S9A), indicating that D118 moved away from the $\beta 3-\beta 4$ turn in the pressure-stabilized intermediate. As the temperature increased, the values of residues 57-58 and 93-94 of the variant increased by approximately 0.2-0.3 (Figure S9B), indicating that residue 118 moved away from the $\beta 3-\beta 4$ and $\beta 6-\beta 7$ turns in the temperature-stabilized intermediate. Similarly, as pressure or temperature increased, the $I$ para $/ I$ dia values of residues $94-97$ of the A140C variant increased by $0.2-0.3$, indicating that residue 140 moved away from the $\beta 7$ in both of the intermediates (Figure S10A and B).I para/I dia values of residues 36, 37, and 106 for the A140C variant are summarized in Figure S11. A pair of cross-peaks was only observed for residue 36. Unlike E128C, the intensity ratio of the new residue 36 peak is similar to that of the original one (Fig. S11A). The ratios at higher temperature were significantly higher for residues 36 and 37, and slightly lower for residue 106 (Fig. $\mathrm{S} 11 \mathrm{~B}$ ), showing opposite tendencies with the data from E128C. In addition, for the variants D118C and A140C, residues 33-39, including the $\beta 1-\beta 2$ turn, maintained lower $I$ para $/ I$ dia values (i.e., $0-0.6$ ) in both intermediates.

These results indicate that the pressure- and temperature-stabilized intermediates shared a common structural feature in which residue 128 moved away from the $\beta 5-\beta 6$ turn (consisting of residues 82 and 83 ) while becoming more accessible to the $\beta 3-\beta 4$ turn region, including residues 59 and 62 . Moreover, residues 118 and 140 moved away from the N-terminal domain, yet remained accessible to the $\beta 1-\beta 2$ turn. As residues 118 , 128 , and 140 are in the turn region between $\beta 8-\beta 9, \beta 9-\beta 10$, and $\beta 10-\beta 11$, respectively, these PRE results suggest that the central $\beta$-sheet, i.e., $\beta 8-\beta 10$, is partially disordered, and thus is located a longer average distance from the N-terminal domain in the intermediates than it is in the native conformation (Figure 1). Alternatively, the $\beta$-sheet region may retain weak specific contact with the $\beta 1-\beta 2$ turn, owing to a twist of the central $\beta$-sheet and increased flexibility of the polypeptide chain. Such specific long-range contact has also been observed in intrinsically disordered proteins such as $\alpha$-synuclein. ${ }^{23,36}$

New peaks appeared in the central aspect of the spectrum, an area in which disordered polypeptide chains are typically observed, as pressure or temperature was increased. The original signals corresponding to the N-terminal domain and the new NMR signals showed gradual decreases in intensity after incubating the protein for a few days at $250 \mathrm{MPa}$, likely owing to protein aggregation. Therefore, the assignment of new peaks using the conventional triple-resonance NMR experiments has not been completed. However, it is useful to analyze the $I$ para $/ I$ dia ratios for new peaks that likely correspond to amide groups in the central and C-terminal domains. Figure 7A shows the frequency of $I$ para $/ I$ dia values for all original cross-peaks that originated from the native protein conformation, as the paramagnetic probe was attached at residue 140 in the central domain. The ratios were widely distributed between 0 and 1; however, were primarily above 0.8 , indicating that a folded conformation remained near the paramagnetic probe, and many amide groups were not accessible to the probe. Figures $7 \mathrm{~B}$ and $\mathrm{C}$ show the frequencies of $I$ para $/ I$ dia values of new peaks in the pressure-stabilized and temperature-stabilized intermediates, respectively, of the A140C variant. In the temperature-stabilized intermediate (Figure $7 \mathrm{C}$ ), the frequencyvs. $I$ para $/ I$ dia plot had a Gaussian shape, with a center value of approximately 0.7 ; frequencies of less than 0.2 and more than 0.9 were not observed, indicating an entirely different shape from the native conformation. Since a Gaussian shape is typically obtained for random events, the PRE results may have resulted from an ensemble of disordered conformations of the polypeptide chain. The PRE provides $1 / r^{6}$ ensemble-averaged distance information, and thus different conformations may show different PRE effects. Even amide groups more than $25 \AA$, apart from the paramagnetic probe in the native conformation, could transiently access the probe in the intermediates due to the increased flexibility of the polypeptide chain, while very few amide groups showed consistently strong PRE effects. Although the $I_{\text {para }} / I_{\text {dia-frequency profile of the pressure-intermediate was }}$ similar to that of the temperature, the Gaussian shape was not as apparent, and the strong and weak $I$ para $/ I$ dia ratios, i.e., $>0.9$ and $<0.2$, were still observed (Figure $7 \mathrm{~B}$ ). Since an intermediate population is retained in both conditions (Figure S2 and Figure S3), the differences in the $I$ para/I dia-frequency profiles indicate that the pressure-intermediate has a more compact conformation. Similar frequency profiles were 
observed for the D118C and E128C variants, as shown in Figures S12 and S13, respectively.

\subsection{Molecular dynamics simulation}

We sought to characterize the locally disordered intermediate conformation of OspA from PRE data. However, a conversion of $R_{2 \mathrm{PRE}}$ into distance is not straightforward in this case since the protein exists as a mixture of different conformational states, including $\sim 80 \%$ locally disordered, $\sim 20 \%$ native, and minimal amounts of completely unfolded protein. Even for the completely unfolded state, it is not easy to reconstruct the probability distribution of the distance between the paramagnetic probe and each amide proton, as multiple sources of stochastic dynamics in the system must be considered. ${ }^{30}$ Here, to produce potential structural models of the intermediate conformations of $\mathrm{WT}^{*}$ OspA, we performed a $1 \mu \mathrm{s}$ MD simulation with the $N V T$ ensemble $(350 \mathrm{~K})$. The $\mathrm{MD}$ simulation demonstrated that temperature-induced disordering of the $\beta$-sheet began at the $\mathrm{C}$-terminal region, followed by the central region.

Distance distributions were calculated between the paramagnetic probe and each amide proton for four different time-regions in the MD trajectory, corresponding to the different levels of disorder in $\beta 9-\beta 11$. Figure S14 presents the distance distribution between the $\mathrm{C}_{\beta}$ of residue 118/128/140 (a substitute for the paramagnetic center) and the amide proton of residue 37 for the folded, partially disordered, and completely disordered ensembles. A specific simulation snapshot was obtained in the folded and partially disordered ensembles, in which $\beta 11, \beta 10$, and $\beta 9$ are disordered (Figure 8A). The distance for the closest approach $d{ }_{0}$ and that of the maximum separation $L$ for the ensembles were obtained from the distance distribution. In the partially disordered and completely disordered ensembles, the distance distribution became wider than that of the folded ensemble. $I$ para $/ I$ dia profiles of the folded and the partially disordered ensembles, as predicted by the MD simulation, are shown in Figure 8B (see Methods). Increasing the ratios for residues 33, 93, and 94 of the D118C variant can be explained by the partially disordered ensemble 3 (PDE3, Figure S8). Meanwhile, the increased ratios of residues 80-83, 103, and 106 of the E128C may have resulted from partially disordered ensembles 1 and 2 (PDE1 and PDE2). Further, PDE1 and PDE2 can explain the incremental ratios for residues 36, 37, 94, and 95 of the A140C variant. However, no ensemble can explain the decreases observed in the ratios of residues $33-37,59$, and 62 . In short, any single specific ensemble cannot explain the entire profile of $I$ para $/ I_{\text {dia. }}$. Moreover, there is no evidence to suggest that the new peaks corresponding to the residues in the central $\beta$-sheet appeared in the central portion of the spectrum. Hence, we do not deny broadening and missing of HSQC cross-peaks owing to conformational dynamics; however, we speculate that the central $\beta$-sheet does not become unfolded, but rather partially disordered, and contains heterogeneous conformations in the intermediates.

Thus, the intermediate state of the protein is likely a mixture of conformations, with different levels of partial disorder in the central $\beta$-sheet. This idea is consistent with the conformational heterogeneity of the equilibrium intermediate mapped by native state hydrogen exchange NMR and scanning mutagenesis. ${ }^{10,11}$ To satisfy the entire profile more quantitatively, PRE should be measured under conditions in which the intermediate state is more dominantly stabilized, and larger numbers of snapshots should be generated via MD simulation of the partially disordered protein.

\subsection{Functional significance of the intermediate conformations}

The current PRE results provide further evidence of disorder in the central and C-terminal regions of the OspA intermediates and similarities between the effects of pressure and temperature on the protein. In addition, a possible ensemble of partially disordered conformations was suggested from the MD simulation. These data indicate that the intermediates more readily expose the tick receptor-binding sites (i.e., residues 236-237 and 242-244) ${ }^{8}$ than does the basic folded conformation. Hence, the transition into the intermediatesin vivo may accelerate the binding of OspA to the tick receptor. Although the folded OspA protein and its C-terminal fragment have been included as vaccine candidates to prevent Borreliatransmission, ${ }^{17}$ the intermediates, in which the central and C-terminal domain are disordered, are unlikely to be recognized by antibodies targeted at the folded C-terminal portion, thereby reducing the effectiveness of the vaccine. The disordering of the C-terminal domain is also considered to be important when the protein is translocated 
through the outer membrane in the C-terminal-to-N-terminal direction. However, destabilization of the C-terminal domain by site-specific mutation induced protein degradation. ${ }^{16}$ Results from the present and previous studies indicate that a delicate interplay between localized conformational dynamics and thermodynamic stability may be required for OspA function.

\section{Conclusion}

Although structural characterization of protein intermediates remains challenging, the partial knowledge that we gathered here not only expands the current understanding on the molecular mechanism of protein function, but also facilitates novel drug design. The intermediate state of OspA consists of a mixture of conformations with different levels of partial disorder in the C-terminal region. The C-terminal region of the intermediate is partially disordered; it however retains weak specific contact with the N-terminal region, owing to a twist of the central $\beta$-sheet and increased flexibility of the polypeptide chain. These intermediates may escape antibody recognition. Information regarding localized conformational dynamics and thermodynamic stability might be useful for the rational design of effective OspA vaccines. Moreover, since PRE observation under high pressure is only limited in ubiquitin, the present is a confirmation of PRE under high pressure. ${ }^{24}$ A combination of PRE-assisted high-pressure NMR and MD simulation is, in general, useful for characterizing the dynamic structures of functionally essential protein intermediates.

\section{ASSOCIATED CONTENT}

Supporting Information Figure S1-S14

\section{Accession codes}

OspA (Borrelia burgdorferi), UniProtKB-Q45040

\section{Author contributions}

The manuscript was written by R. K. NMR measurements and analyses were performed by T. W., S. K., and R. K. MD simulations were performed by T. K. All authors reviewed and approved the final manuscript.

\section{Notes}

The authors declare no competing interests.

\section{REFERENCES}

1. Boehr DD, McElheny D, Dyson HJ, and Wright PE. The dynamic energy landscape of dihydrofolate reductase catalysis. Science. 2006;313:1638-1642.

2. Boehr DD, Dyson HJ, and Wright PE. An NMR perspective on enzyme dynamics. Chem Rev. 2006;106:3055-3079.

3. Booth DR, Sunde M, Bellotti V, Robinson CV, Hutchinson WL, Fraser PE, Hawkins PN, Dobson CM, Radford SE, Blake CCF, and Pepys MB. Instability, unfolding and aggregation of human lysozyme variants underlying amyloid fibrillogenesis. Nature. 1997;385:787-793.

4. Chiti F and Dobson CM. Amyloid formation by globular proteins under native conditions. Nat Chem Biol. 2009;5:15-22.

5. Li H, Dunn JJ, Luft BJ, and Lawson CL. Crystal structure of Lyme disease antigen outer surface protein A complexed with an Fab. Proc Natl Acad Sci U S A. 1997;94:3584-3589.

6. Makabe K, Tereshko V, Gawlak G, Yan S, and Koide S. Atomic-resolution crystal structure of Borrelia burgdorferi outer surface protein A via surface engineering. Protein Sci. 2006;15:1907-1914.

7. Pal U, Li X, Wang T, Montgomery RR, Ramamoorthi N, deSilva AM, BaoFK, Yang XF, Pypaert M, Pradhan D, Kantor FS, Telford S, Anderson JF, and Fikrig E. TROSPA. an Ixodues scapularis receptor for Borrelia burgdorferi. Cell. 2004;119:457-468.

8. Pal U, de Silva AM, Montgomery RR, Fish D, Anguita J, Anderson JF, Lobet Y and Fikrig E. Attachment of Borrelia burgdorferi within Ixodes scapularis mediated by outer surface protein A. J Clin Invest. 2000;106:561-569. 
9. Pham TN, Koide A, and Koide SA. stable single-layer beta-sheet without a hydrophobic core. Nat Strct Biol. 1998;5:115-119.

10. Yan S, Kennedy SD and Koide S. Thermodynamic and kinetic exploration of the energy landscape of Borrelia burgdorferi OspA by native state hydrogen exchange. J Mol Biol. 2002;323:363-375.

11. Yan S, Gawlak G, Smith J, Silver L, Koide A and Koide S. Conformational heterogeneity of an equilibrium folding intermediate quantified and mapped by scanning mutagenesis. J Mol Biol. 2004;338:811825 .

12. Koide S, Bu ZM, Risal D, Pham TN, Nakagawa T, Tamura A and Engelman DM. Multistep denaturation of Borrelia burgdorferi OspA, a protein containing a single-layer beta-sheet. Biochemistry. 1999;38:4757-4767.

13. Nakagawa T, Shimizu H, Link K, Koide A, Koide S and Tamura A. Calorimetric dissection of thermal unfolding of OspA, a predominantly beta-sheet protein containing a single-layer beta-sheet. J Mol Biol. 2002;323:751-762.

14. Kitahara R, Simorellis AK, Hata K, Maeno A, Yokoyama S, Koide S and Akasaka K. A delicate interplay of structure, dynamics, and thermodynamics for function: a high pressure NMR study of outer surface protein A. Biophys J. 2012;102:916-926.

15. Makabe K, Nakamura T, Dhar D, Ikura T, Koide S and Kuwajima K. An Overlapping Region between the Two Terminal Folding Units of the Outer Surface Protein A (OspA) Controls Its Folding Behavior. J Mol Biol. 2018;430:1799-1813.

16. Schulze RJ, Chen S, Kumru OS and Zückert WR. Translocation of Borrelia burgdorferi surface lipoprotein OspA through the outer membrane requires an unfolded conformation and can initiate at the C-terminus. Mol Microbiol. 2010;76:1266-1278.

17. Koide S, Yang X, Huang X, Dunn JJ and Luft BJ. Structure-based design of a second-generation Lyme disease vaccine based on a C-terminal fragment of Borrelia burgdorferi OspA. J Mol Biol. 2005;350:290299.

18. Livey I, O'Rourke M, Traweger A, Savidis-Dacho H, Crowe BA, Barrett PN, Yang X, Dunn JJ, and Luft BJ. A new approach to a Lyme disease vaccine. Clinical Infectious Diseases. 2011;52:S266-S270.

19. Nigrovic LE and Thompson KM. The Lyme vaccine: a cautionary tale. Epidemiol. Infect. 2007;135:1-8.

20. Clore GM and Iwahara J. Theory, practice, and applications of paramagnetic relaxation enhancement for the characterization of transient low-population states of biological macromolecules and their complexes. Chem Rev. 2009;109:4108-4139.

21. Liu Z, Gong Z, Jiang WX, Yang J, ZhuWK, Guo DC, Zhang WP, Liu ML and Tang C. Lys63-linked ubiquitin chain adopts multiple conformational states for specific target recognition. eLife. 2015;4:e05767.

22. Battiste JL and Wagner G. Utilization of site-directed spin labeling and high-resolution heteronuclear nuclear magnetic resonance for global fold determination of large proteins with limited nuclear overhauser effect data. Biochemistry. 2000;39:5355-5365.

23. Salmon L, Nodet G, Ozenne V, Yin GW, Jensen MR, Zweckstetter M and Blackledge M. NMR Characterization of Long-Range Order in Intrinsically Disordered Proteins. J Am Chem Soc. 2010;132:84078418 .

24. Wakamoto T, Ikeya T, Kitazawa S, Baxter NJ, Williamson MP and Kitahara R. Paramagnetic relaxation enhancement-assisted structural characterization of a partially disordered conformation of ubiquitin. Protein Sci. 2019;28:1993-2003.

25. Dunn JJ., Lade BN and Barbour AG. Outer surface protein a (OspA) from the lyme disease spirochete, borrelia burgdorferi: High level expression and purification of a soluble recombinant form of OspA. Protein Exp Purif. 1990;1:159-168.

26. Delaglio F, Grzesiek S, Vuister GW, Zhu G, Pfeifer J. and Bax A. Nmrpipe - a Multidimensional Spectral Processing System Based on Unix Pipes. J Biol NMR. 1995;6:277-293.

27. Johnson BA and Blevins RA. Nmr View - a Computer-Program for the Visualization and Analysis of Nmr Data. J Biol NMR. 1994;4:603-614.

28. Kosen PA. Spin labeling of proteins. Methods Enzymol. 1989;177:86-121.

29. Pawley NH, Koide S and Nicholson L. K. Backbone dynamics and thermodynamics of Borrelia outer 
surface protein A. J Mol Biol. 2002;324:991-1002.

30. Xue Y, Podkorytov IS, Rao DK, Benjamin N, Sun H and Skrynnikov NR. Paramagnetic relaxation enhancements in unfolded proteins: Theory and application to drkN SH3 domain. Protein Sci. 2009;18:1401-1424.

31. Maier JA, Martinez C, Kasavajhala K, Wickstrom L, Hauser KE, Simmerling C. ff14SB: Improving the accuracy of protein side chain and backbone parameters from ff99SB. J Chem Theory Comput. 2015;11:3696-3713.

32. Mongan J, Simmerling CA. McCammon JA, Case D, Onufriev A, Generalized born with a simple, robust molecular volume correction. J Chem Theory Comput. 2007;3:156-169.

33. Pettersen EF, Goddard TD, Huang CC, Couch GS, Greenblatt DM, Meng EC and Ferrin TE. UCSF chimera - A visualization system for exploratory research and analysis. J Comput Chem. 2004;25:16051612 .

34. Tian W, Chen C, Lei X., Zhao J. and Liang J. CASTp 3.0: computed atlas of surface topography of proteins. Nucleic Acids Res. 2018;46:W363-W367.

35. Kawamura T, Wakamoto T, Kitazawa S, Sakuraba S, Kameda T and Kitahara R, Analysis of $\mathrm{O}_{2}{ }^{-}$ binding sites in proteins using gas-pressure NMR spectroscopy: Outer surface protein A. Biophys J. 2017;112:1820-1828.

36. Bernado P, Bertoncini CW, Griesinger C, Zweckstetter M and Blackledge M. Defining long-range order and local disorder in native alpha-synuclein using residual dipolar couplings. J Am Chem Soc. 2005;127:17968-17969.

Figure legends

Figure 1. Three-dimensional structure of OspA. Residues substituted to cysteine and with the attached spin-labeled MTSL are depicted as spheres. OspA contains the N-terminal ( $\beta 1-\beta 7)$ and C-terminal ( $\beta 11$ to the C-terminus) globular domains, which are connected by a single-layer $\beta$-sheet $(\beta 8-\beta 10)$. The image was prepared using Chimera. ${ }^{33}$ Internal cavities were detected using CASTp server with $1.4 \AA$ radius probe. ${ }^{34}$

Figure $2 .{ }^{1} \mathrm{H} /{ }^{15} \mathrm{~N}-\mathrm{HSQC}$ spectra of the reduced OspA E128C variant form as pressure was gradually increased from 0.1 to $250 \mathrm{MPa}$ at $313 \mathrm{~K}$ (black, $0.1 \mathrm{MPa}$; blue, $50 \mathrm{MPa}$; cyan, $100 \mathrm{MPa}$; green, $150 \mathrm{MPa}$; purple, 200 $\mathrm{MPa}$; red, $250 \mathrm{MPa}$ ). (Inset) Change in chemical shifts of V199 (not visible in the main plot).

Figure 3. Resonance intensities (i.e. peak volumes) of the OspA E128C variant at different pressures and temperature conditions. (A) Resonance intensities at $250 \mathrm{MPa}$ relative to those at $0.1 \mathrm{MPa}(313 \mathrm{~K})$ as a function of residue number. (B) Resonance intensities at $318 \mathrm{~K}$ relative to those at $303 \mathrm{~K}(0.1 \mathrm{MPa})$ as a function of residue number.

Figure 4. Intensity ratios, $I$ para $/ I_{\text {dia }}$, for each amide group of the E128C variant for each residue. (A) Pressure dependence of the $I_{\text {para }} / I_{\text {dia }}$ value at $313 \mathrm{~K}$. (B) Temperature dependence of the $I_{\text {para }} / I_{\text {dia }}$ value at $0.1 \mathrm{MPa}$. Secondary structures are indicated at the top of the panels. Error bars for $I$ para $/ I$ dia were estimated from the noise considering error propagation, i.e. $\left(\frac{I_{\mathrm{Para}}}{I_{\mathrm{dia}}}\right)=\left(\frac{I_{\mathrm{para}}}{I_{\mathrm{dia}}}\right) \sqrt{\left(\frac{I_{\mathrm{para}}}{I_{\mathrm{para}}}\right)^{2}+\left(\frac{I_{\mathrm{dia}}}{I_{\mathrm{dia}}}\right)^{2}}$.

Figure 5. Pressure and temperature dependence of peak height ratios of a paramagnetic sample (E128C) to those of a diamagnetic sample, i.e., $I$ para $/ I_{\text {dia. }}$ (A) Locations of residues $59,62,82,83$, and 128 in OspA. (B) Pressure-induced changes in the ratios of residues 59, 82, and 83 at $313 \mathrm{~K}$. (C) Temperature-induced changes in the ratios of residues 59, 62, 82, and 83 at $0.1 \mathrm{MPa}$. Panel A was prepared using Chimera. ${ }^{33}$

Figure 6. Pressure- and temperature-dependent effects on selected HSQC cross-peaks. (A) Selected ${ }^{1} \mathrm{H} /{ }^{15} \mathrm{~N}$ HSQC spectral regions for the reduced MTSL-E128C variant at multiple pressures (black, $0.1 \mathrm{MPa}$; blue, $50 \mathrm{MPa}$; cyan, $100 \mathrm{MPa}$; green, $150 \mathrm{MPa}$; purple, $200 \mathrm{MPa}$; red, $250 \mathrm{MPa}$ ). (B) Selected ${ }^{1} \mathrm{H} /{ }^{15} \mathrm{~N}$ HSQC spectral regions of the protein at multiple temperatures (black, $303 \mathrm{~K}$; blue, $308 \mathrm{~K}$; green, $313 \mathrm{~K}$; red, 318 K). Assignments for G36 and G106 peaks are depicted at the top of the 0.1 MPa-peak in panel A or 303 K-peak in panel B. The peaks of G36 and G106 are marked with asterisks. (C) $I$ para/ $I$ dia values of original 
and new peaks of residues 36, 37, and 106 at $200 \mathrm{MPa}$. (D) $I$ para $/ I$ dia values of the averaged peaks of residues 36,37 , and 106 at 303 and $318 \mathrm{~K}$.

Figure 7. Frequency of $I$ para $/ I$ dia values calculated from HSQC cross-peaks for the A140C variant. (A) Frequency of $I$ para $/ I_{\text {dia }}$ of original cross-peaks at $0.1 \mathrm{MPa}$ and $303 \mathrm{~K}$, corresponding to the native state. (B) Frequency of $I$ para $/ I$ dia of new cross-peaks at $250 \mathrm{MPa}$ and $303 \mathrm{~K}$, corresponding to the pressure-stabilized intermediate. (C) Frequency of $I_{\text {para }} / I_{\text {dia }}$ of new cross-peaks at $0.1 \mathrm{MPa}$ and $318 \mathrm{~K}$, corresponding to the temperature-stabilized intermediate. Broken lines depict value 1.

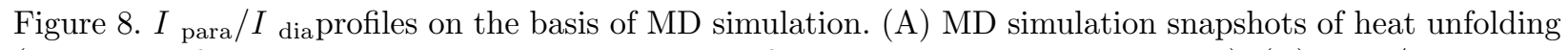
(snapshots after $0.2 \mathrm{~ns}, 62.7 \mathrm{~ns}, 73.1 \mathrm{~ns}$, and $80.7 \mathrm{~ns}$ from top to bottom, respectively). (B) $I$ para $/ I_{\text {dia }}$ ratios for the conformational ensembles of different levels of disorder in $\beta 9-\beta 11$ (black, the folded ensemble (16 snapshots in the MD trajectory of $0.1 \mathrm{~ns} \sim 1.6 \mathrm{~ns}$ ); red, the partially disordered ensemble 1 (PDE1, 31 snapshots in the MD trajectory of $60 \mathrm{~ns} \sim 63 \mathrm{~ns}$ ); blue, the partially disordered ensemble 2 (PDE2, 121 snapshots in the MD trajectory of $63.1 \mathrm{~ns}^{\sim} 75.1 \mathrm{~ns}$ ); green, the partially disordered ensemble 3 (PDE3, 65 snapshots in the MD trajectory of $\left.\left.75.2 \mathrm{~ns}^{\sim} 81.6 \mathrm{~ns}\right)\right) . I$ para $/ I$ dia ratios are calculated from the distance distribution between the $\mathrm{C}_{\beta}$ of residue 118 (top)/128 (middle)/140 (bottom) (a substitute for the paramagnetic center) and the amide proton of each residue with $\tau_{\mathrm{r}}$ of $12 \mathrm{~ns}$ (see Fig. S14). Panel A was prepared using Chimera. ${ }^{33}$

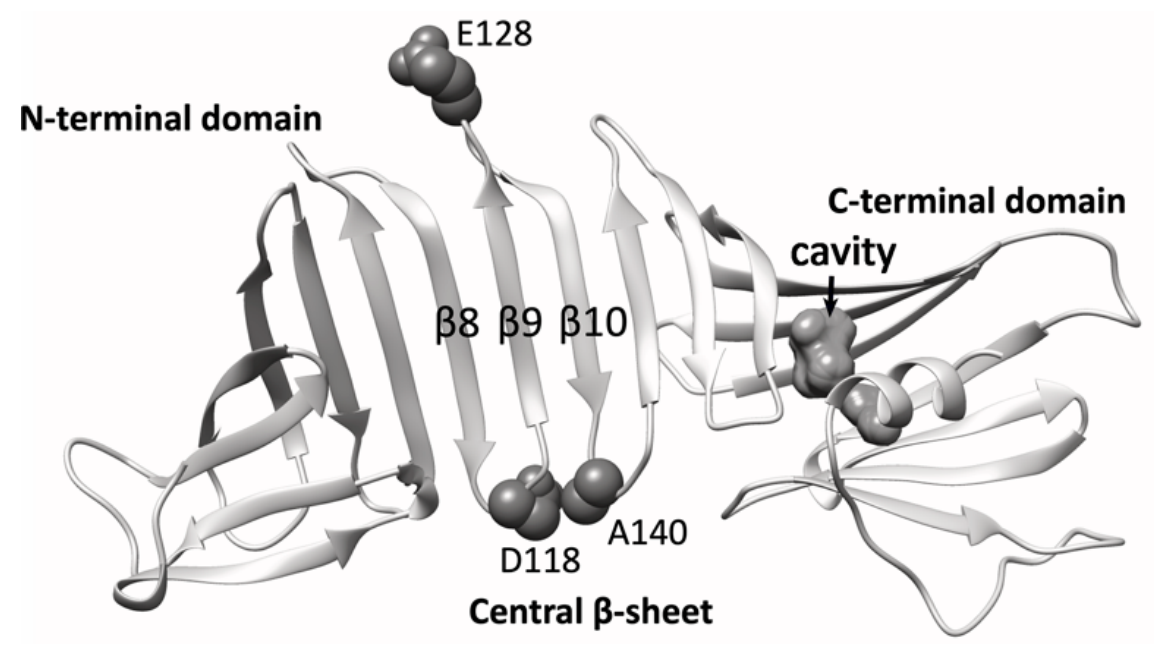

Figure 1 


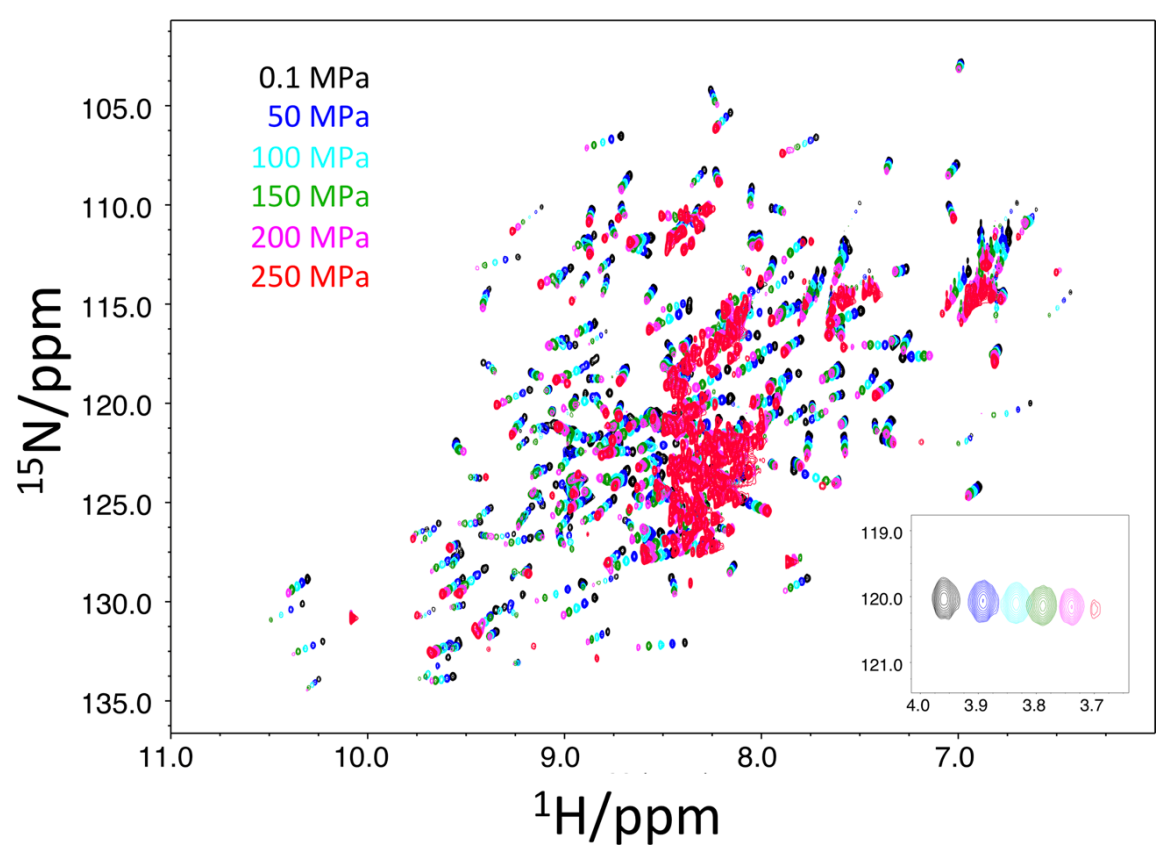

Figure 2
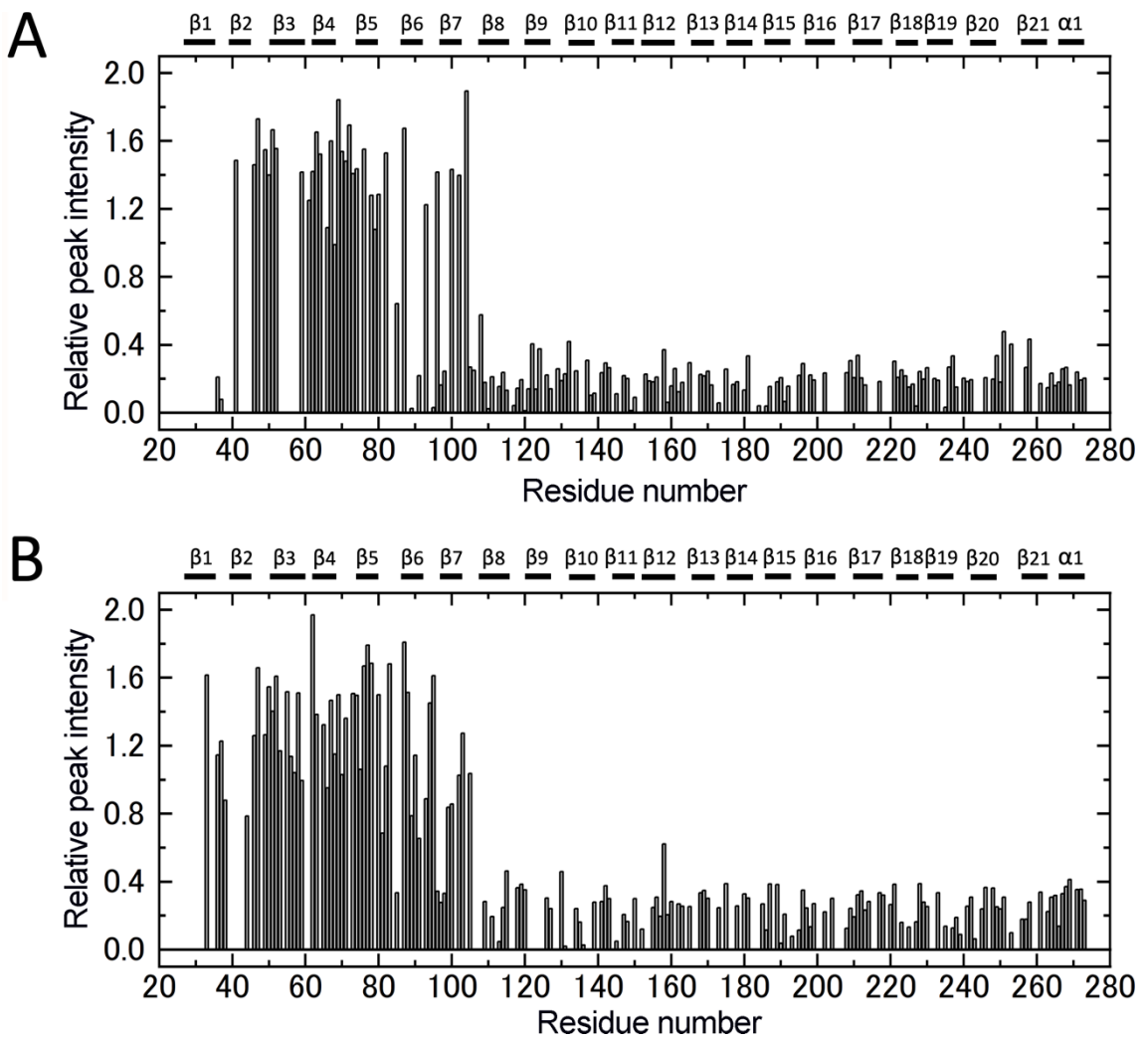

Figure 3 

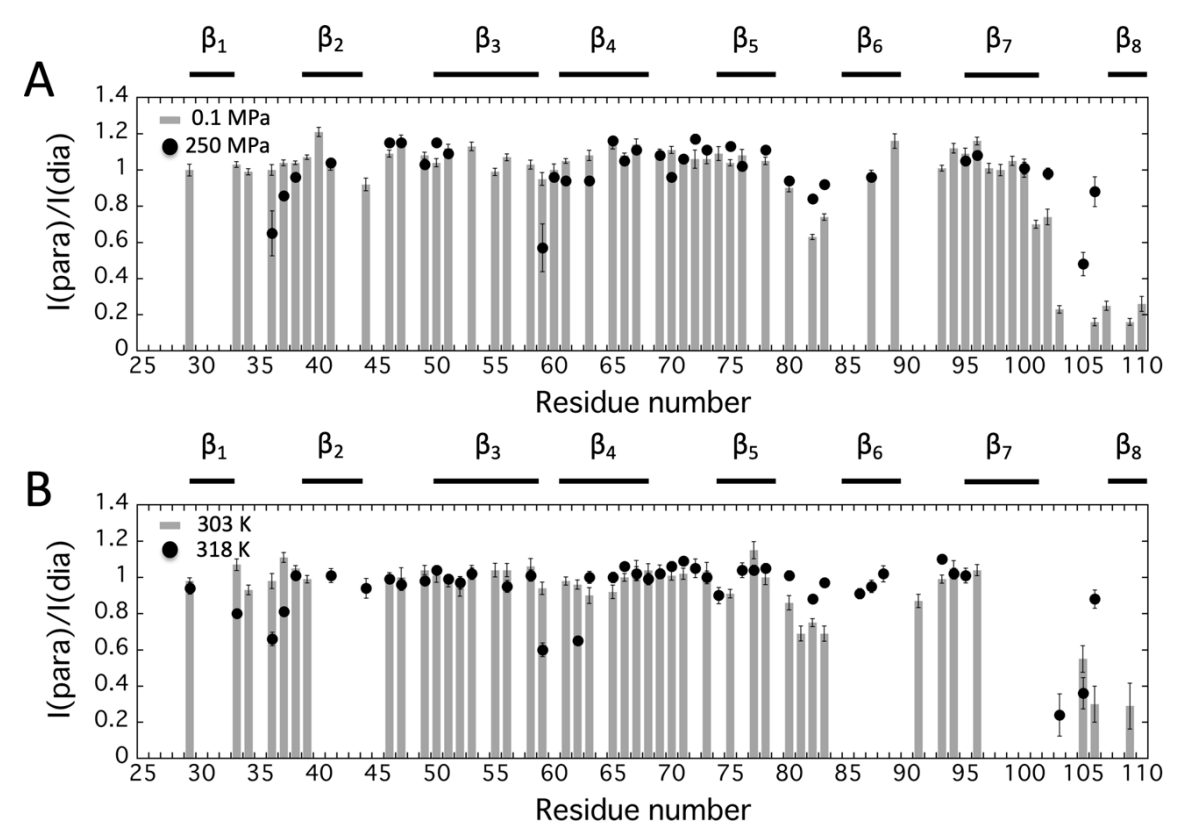

Figure 4 

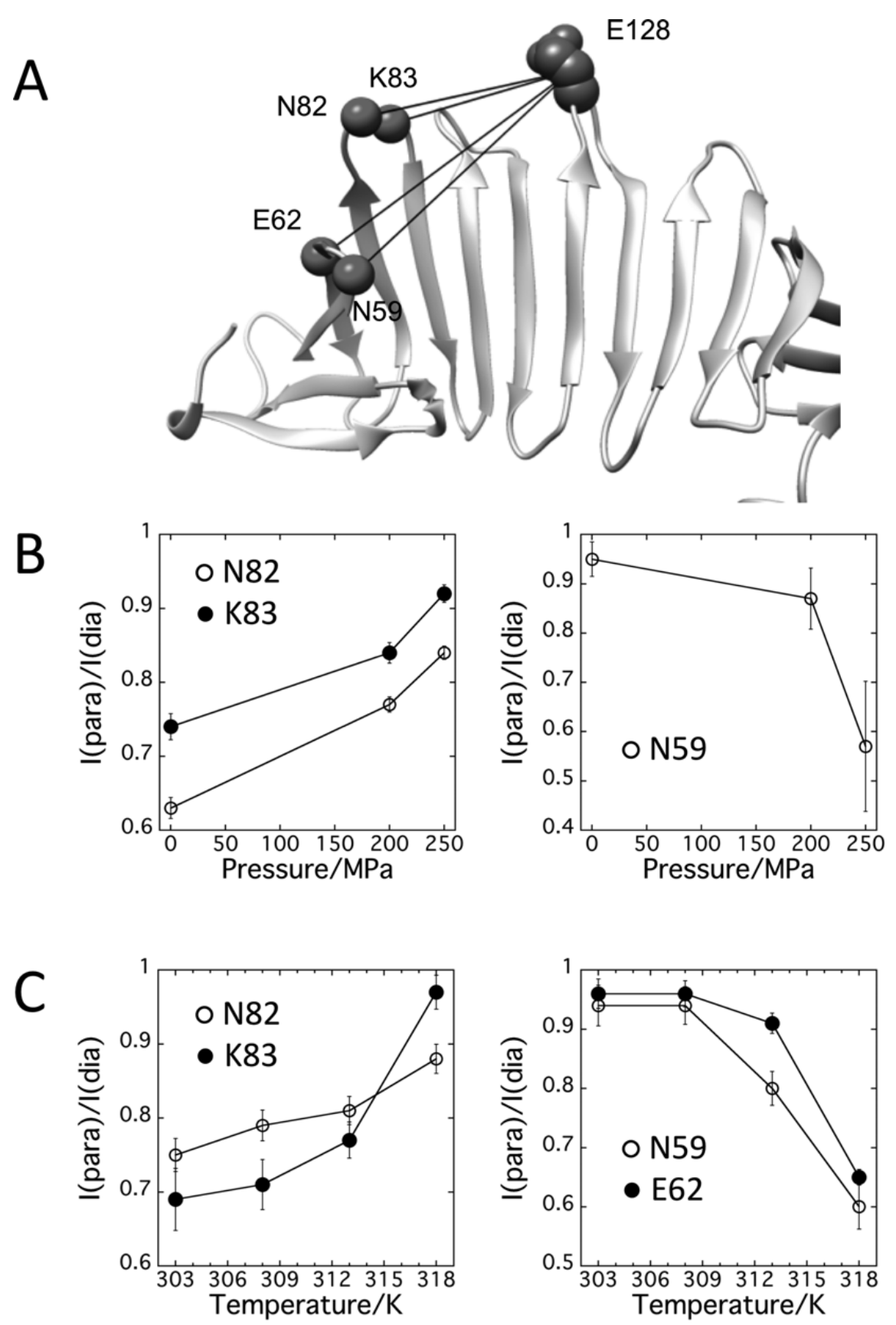

Figure 5 

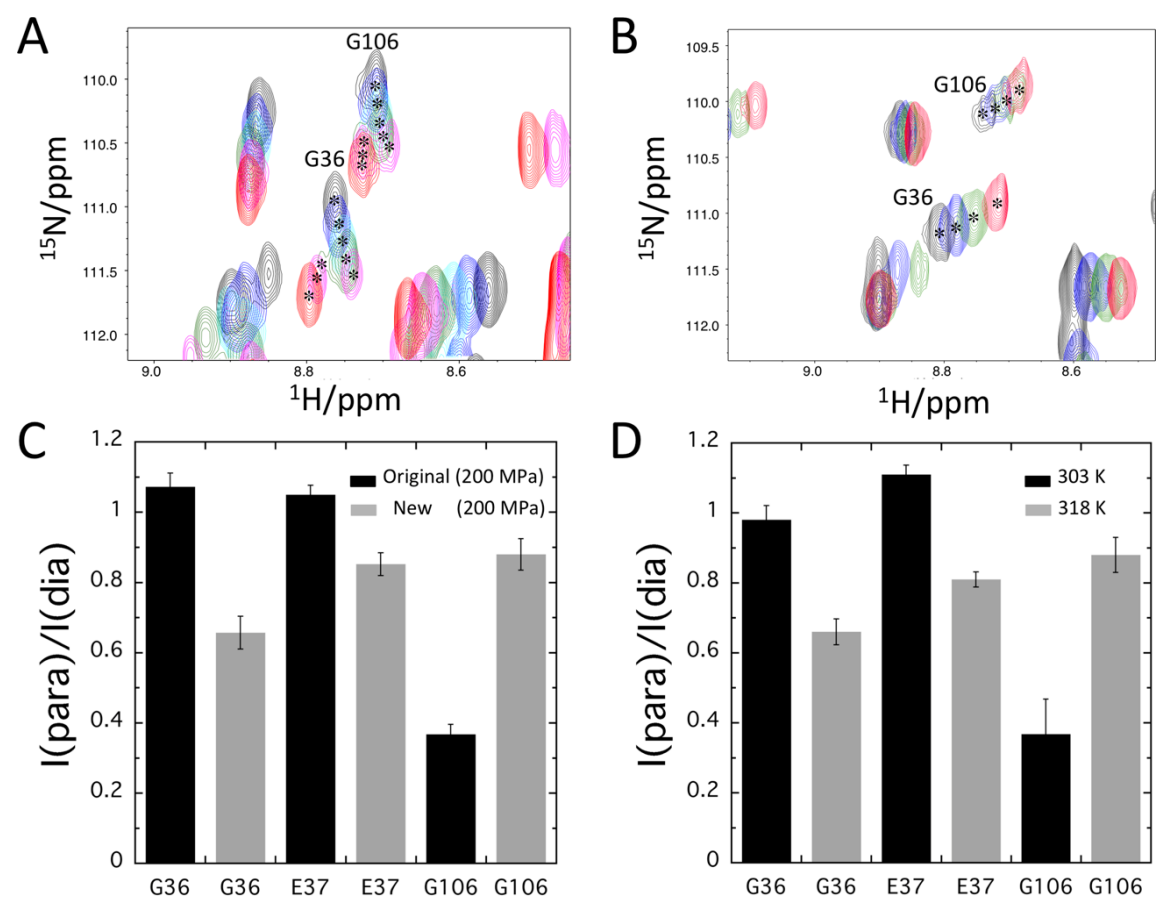

Figure 6
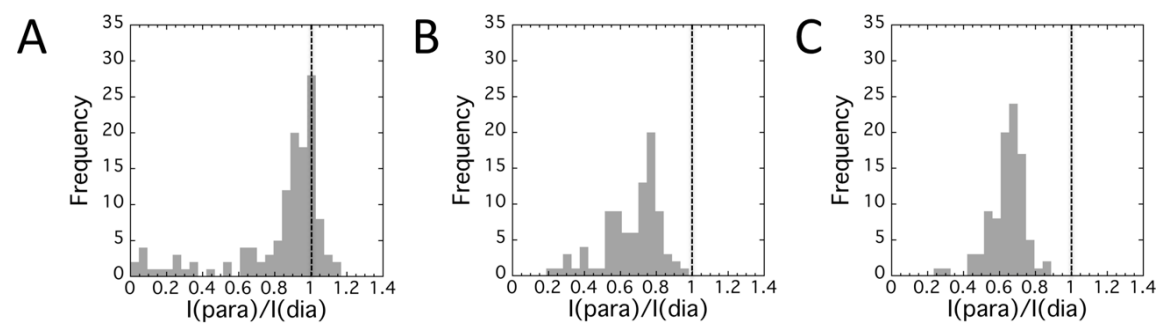

Figure 7 

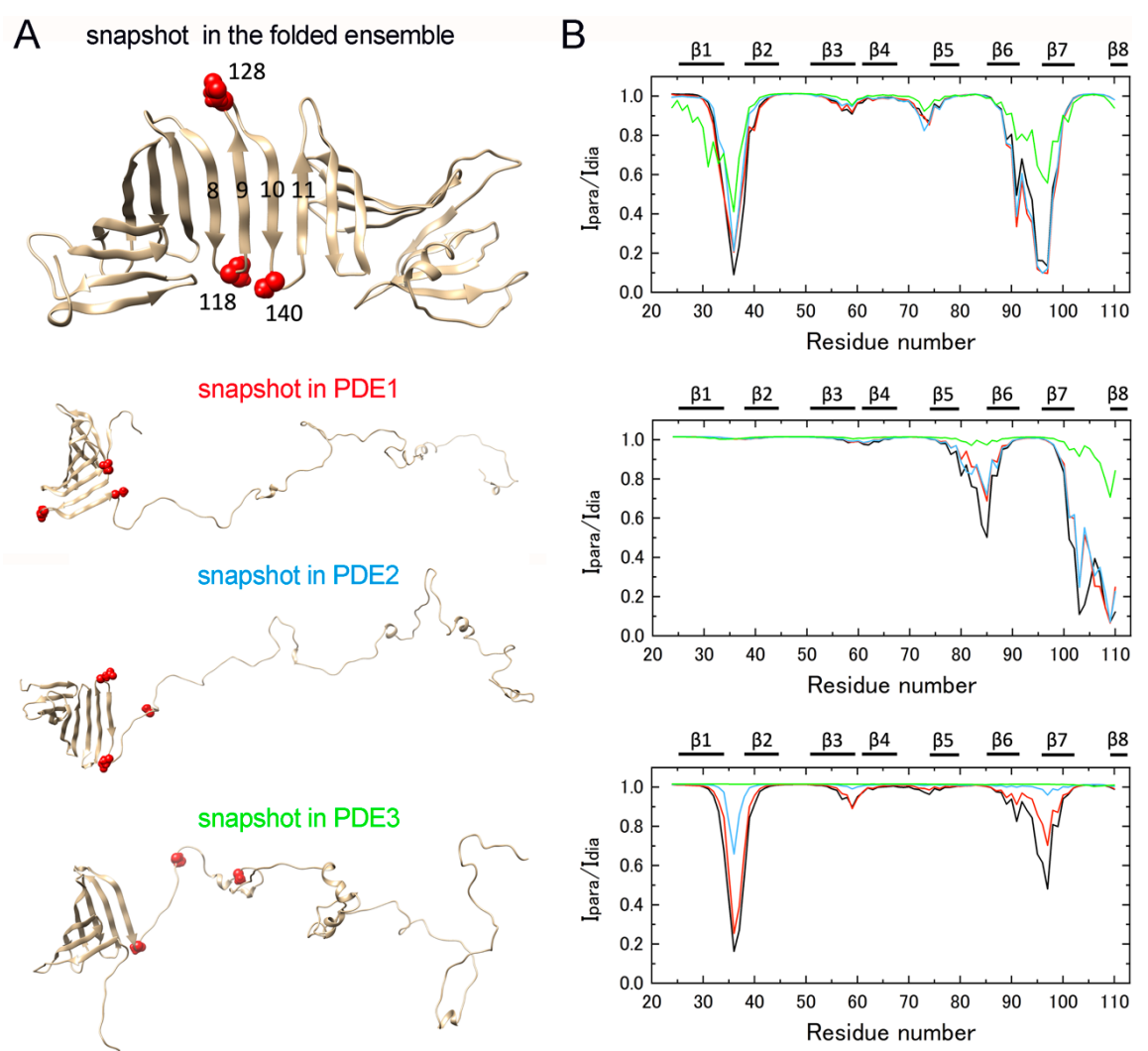

Figure 8 